\title{
ON THE RELATIONSHIP BETWEEN ORDINARY THIN SETS AND FULL-THIN SETS
}

\author{
J. S. HWANG and H. L. JACKSON
}

(Received 5 March; revised 12 November 1979)

Communicated by E. Strzelecki

\begin{abstract}
In this work we demonstrate that if $\Omega \subset \mathbf{R}^{n}(n \geqslant 3$ ) is either a half space or a unit ball, and if $E \subset \Omega$ then $E$ is an ordinary thin set at a boundary point of $\Omega$ (including the point at infinity if $\Omega$ is a half space) if and only if it is a full-thin set at the corresponding Kuramochi boundary point of $\Omega$. The case for $n=2$ has already been considered in an earlier work.
\end{abstract}

1980 Mathematics subject classification (Amer. Math. Soc.) : 31 B 05.

\section{Introduction}

In an earlier paper we noticed that ordinary thin sets are exactly the same as full (or Kuramochi)-thin sets with relation to any boundary point of the unit disk or half plane, but that these two kinds of thin sets are in general noncomparable for a Jordan region (see Hwang and Jackson (1978), p. 444). Because of the theory of double Riemann surfaces it is easy to describe the Kuramochi kernel (modulo a deleted compact disk $K_{0}$ ) in terms of the Green kernel of the double surface (see Constantinescu and Cornea (1963)) and comparable results can be obtained for fullsuperharmonic functions (see Maeda, Ohtsuka et al. (1968), p. 4, 5, and 28). In higher dimensions, however, it appears to be necessary to employ slightly different techniques in order to prove that ordinary thin sets coincide with full-thin sets at any boundary point of a half space including the point at infinity, or of the unit ball. In fact we found it essential to use somewhat different methods for the ball as opposed to the half space, although we were able to define Green potentials in the doubled region in both cases which extended the full-superharmonic functions in the original region. The reader is referred to Brelot (1971), p. 54, for basic properties of ordinary

This research was partially supported by NSERC Grant No. A7322. 
thin sets, and to Hwang and Jackson (1978), p. 442, 443, for full-thin sets, and the Kuramochi kernel function.

\section{The half space $\Omega$ in $\mathbf{R}^{n}(n \geqslant 3)$}

For a half space $\Omega=\left\{x=\left(x_{1}, \ldots, x_{n}\right): x_{1}>0\right\}$ the technique of doubling the region about its boundary $\partial \Omega=\left\{x: x_{1}=0\right\}$ will give positive results provided that special attention is given to the point at infinity. As usual we delete a compact ball $K_{0}$ from $\Omega$ to obtain $\Omega-K_{0}=\Omega_{0}$ and let $K_{0}^{*}$ (respectively $\Omega_{0}^{*}$ ) be the reflection of $K_{0}$ (respectively $\Omega_{0}$ ) about $\partial \Omega$. If $a \in \Omega_{0} \cup \partial \Omega$ and $a *$ is the reflection of $a$ about $\partial \Omega$ we shall demonstrate that the Kuramochi function for $\Omega_{0}$ with pole at $a$ is of the form $N_{a}$ $=S_{a \Omega_{0 \cup \partial \Omega}}$ where $S_{a}=\widetilde{G}_{a}+\widetilde{G}_{a^{*}}+\lambda_{a} W$ given that $\widetilde{G}_{a}$ (respectively $\widetilde{G}_{a^{*}}$ ) is the ordinary Green function for the doubled region $\Omega_{0}=\Omega_{0} \cup \partial \Omega \cup \Omega_{0}^{*}=\mathbf{R}^{n}-\left(K_{0} \cup K_{0}^{*}\right)$ with pole at $a$ (respectively $a *), \lambda_{a}>0$ is a constant depending on $a$ to be determined, and $W$ is the harmonic measure of $\{\infty\}$ with respect to the region $\Omega_{0}$. We recall (see Hwang and Jackson) in the case where $n=2, S_{a}=\widetilde{G}_{a}+\widetilde{G}_{a^{*}}$ in both the half plane and unit disk. When $n \geqslant 3$, however, we shall see that the term involving $W$ cannot be omitted for a half space. For any $a \in \Omega_{0} \cup \partial \Omega$ we shall choose $\lambda_{a}>0$ (uniquely) in order to ensure that $S_{a}$ extended to $\mathbf{R}^{n} \cup\{\infty\}$ will in fact be harmonic at $\infty$ in the sense of Brelot (1944), p. 309.

Following Brelot (1944), p. 302, we shall let $\varphi_{n}$ be the flux of the fundamental function over any sphere that contains the pole in its interior, and observe that $\varphi_{n}$ equals $(n-2)$ times the surface measure of the unit sphere in $\mathbf{R}^{n}$.

LEMMA 2.1. For any $a \in \Omega_{0} \cup \partial \Omega, S_{a}=\widetilde{G}_{a}+\widetilde{G}_{a^{*}}+\lambda_{a}$ Wis harmonic at $\infty$ if and only if $\lambda_{a}=2 \varphi_{n} W(a)\|W\|^{-2}$ where $\|W\|^{2}$ is the Dirichlet integral of $W$ over $\widetilde{\Omega}_{0}$.

ProOF. Let $B$ be any ball of centre 0 such that its radius $r>|a|$ and $K_{0} \subset$ int $B$. The flux of $S_{a}$ over $\partial B$ is constant with respect to $r$ and equals the sum of the fluxes over $\partial B$ of the component functions. It will be sufficient to prove that $S_{a}$ is harmonic at $\infty$ if and only if the flux of $S_{a}$ always vanishes over $\partial B$ (see Brelot (1944), p. 309). Since $W$ is harmonic on $\Omega_{0}$ an application of the Green formulae indicates that the flux of $W$ over $\partial B$ equals the flux of $W$ over $\partial K_{0} \cup \partial K_{0}^{*}$. Let $\hat{R}_{1}^{K_{0} \cup \mathbf{K}_{0}^{*}}$ be the regularized reduced function of 1 relative to $K_{0} \cup K_{0}^{*}$ in $\mathbf{R}^{n}$ (see Helms (1969), p. 135). If we choose a suitable increasing sequence of balls whose union is all of $\mathbf{R}^{n}$ an elementary argument involving the use of Green identities can be applied to obtain the result that the energy of the potential $\hat{R}_{1}^{\boldsymbol{K}_{0} \cup \boldsymbol{K}_{0^{*}}}$ equals the flux of $W$ over $\partial B$ which in turn equals the ordinary capacity of $K_{0} \cup K_{0}^{*}$ in $\mathbf{R}^{n}$. All of these quantities are also equal to $\|W\|^{2}=\left\|R_{1}^{K_{0} \cup K_{0^{*}}}\right\|^{2}$ where $\|W\|^{2}$ is the Dirichlet integral of $W$ over $\widetilde{\Omega}_{0}$. If $\bar{B}_{\varepsilon}(a)=$ $\left\{x \in \mathbf{R}^{n}:|x-a| \leqslant \varepsilon\right\} \subset \boldsymbol{\Omega}_{0} \cap B$ then the flux of $\widetilde{G}_{a}$ over $\partial B$ equals the flux of $\widetilde{G}_{a}$ over $\partial B_{\varepsilon}(a)+$ the flux of $\tilde{G}_{a}$ over $\partial K_{0} \cup \partial K_{0}^{*}$. The flux of $\widetilde{G}_{a}$ over $\partial B_{\varepsilon}(a)$ is $-\varphi_{n}$ and the flux 
of $\tilde{G}_{a}$ over $\partial K_{0} \cup \partial K_{0}^{*}$ is $\varphi_{n} \hat{R}_{1}^{K_{0}} \cup K_{0^{*}}(a)$ so that $-\varphi_{n} W(a)$ is the flux of $\tilde{G}_{a}$ over $\partial B$. A similar result can be obtained for $\widetilde{G}_{a^{*}}$ and since $W(a *)=W(a)$ we obtain the resul that the flux of $S_{a}$ over $\partial B$ is $\lambda_{a}\|W\|^{2}-2 \varphi_{n} W(a)$. The lemma follows. //

REMARK 2.1. $S_{a}$ is superharmonic (respectively subharmonic) at $\infty$ if and only i $\lambda_{a} \geqslant 2 \varphi_{n} W(a)\|W\|^{-2}$ (respectively $\lambda_{a} \leqslant 2 \varphi_{n} W(a)\|W\|^{-2}$ ).

THEOREM 2.1. If $a \in \Omega_{0}=\Omega-K_{0}$ the Kuramochi function for $\Omega_{0}$ with pole at $a$ is $N_{a}(x)=\tilde{G}_{a}(x)+\widetilde{G}_{a^{*}}(x)+2 \varphi_{n} W(a) W(x)\|W\|^{-2}$ for $x \in \Omega_{0} \cup \partial \Omega$.

PROOF. Let $S_{a}(x)=\widetilde{G}_{a}(x)+\widetilde{G}_{a^{*}}(x)+\lambda_{a} W(x)$ be defined on $\Omega_{0}$ as in Lemma 2.1. II $K \subset \Omega$ is compact such that $K_{0} \cup\{a\} \subset$ interior $K$ such that the divergence theorem is applicable to $(\Omega-K) \cap B$ for sufficiently large balls $B$, and if $u \in H D(\Omega-K)$ which has a continuous extension onto $\partial K$ which coincides with $S_{a \mid \partial K}$ then it is sufficient to prove that $\left\|S_{a}\right\|_{\Omega-K} \leqslant\|u\|_{\Omega-K}$. The fact that $S_{a \Omega-K} \in H D(\Omega-K)$ follows from the fact that $W \in H D\left(\widetilde{\Omega}_{0}\right)$ and that $\tilde{G}_{a \mid \Omega-K}$ and $\tilde{G}_{a * \mid \Omega-K}$ are both restrictions to $\Omega-K$ of $\tilde{G}$ potentials with finite energy. If $u=S_{a}+V$ then $\|u\|_{\Omega-K}^{2}=\|V\|_{\Omega-K}^{2}+\| S_{a}$ $\|_{\Omega-K}^{2}+2\left(S_{a}, V\right)_{\Omega-K}$ where $V \in H D(\Omega-K)$ such that $\lim _{x \rightarrow x^{*}, x \in \Omega-K} V(x)=0$ for every $x * \epsilon \partial K$. It is now sufficient to prove that $\left(S_{a}, V\right)_{\Omega-K}=0$ for $\lambda_{a}$ chosen to be $2 \varphi_{n} W(a)\|W\|^{-2}$. If $B$ is a sufficiently large ball of radius $r$ an application of Green's first identity together with an exhaustion argument indicates that

$$
\left(S_{a}, V\right)_{\Omega \cap B-K}=\int_{\Omega \cap \partial B} V\left(\frac{\partial S_{a}}{\partial v}\right) d \sigma_{n-1}
$$

because $V \equiv 0$ on $\partial K$ and $\partial S_{a} / \partial v \equiv 0$ on $\partial \Omega$. Therefore

$$
\left(S_{a}, V\right)_{\Omega-K}=\lim _{r \rightarrow \infty} \int_{\Omega \cap \hat{\theta} B} V\left(\frac{\partial S_{a}}{\partial v}\right) d \sigma_{n-1} .
$$

Since $V \in H D(\Omega-K), V$ has a limit along almost every Green line issuing from $a$ (see Maeda (1964)). For those Green lines that approach infinity $V$ has a common limit $L$, necessarily finite, called the normal extension of $V$ at $\infty$ by Brelot (1953), pp. 407-408. Furthermore,

$$
\lim _{r \rightarrow \infty} \int_{\hat{B} B} \frac{|V-L| d \sigma_{n-1}}{r^{n-1}}=0
$$

which in turn implies that

$$
\lim _{r \rightarrow \infty} \int_{\partial B}|V-L|\left(\frac{\partial S_{a}}{\partial v}\right) d \sigma_{n-1}=0
$$


(see also Brelot (1953), p. 394, Théorème 5). Therefore

$$
\left(S_{a}, V\right)_{\Omega-K}=\frac{L}{2}\left(\text { flux of } S_{a} \text { over } \partial B\right)
$$

which vanishes by the proof of Lemma 2.1 if we choose $\lambda_{a}=2 \varphi_{n} W(a)\|W\|^{-2}$. Our theorem follows. //

REMARK 2.2. If $H D_{0}\left(\Omega_{0}\right)$ is the closed subspace of $H D\left(\Omega_{0}\right)$ consisting of those harmonic functions that vanish continuously on $\partial K_{0}$, then there exists $U_{a} \in H D_{0}\left(\Omega_{0}\right)$ defining a bounded linear functional on $H D_{0}\left(\Omega_{0}\right)$ such that $\left(U_{a}, u\right)=\varphi_{n} u(a)$ for every $u \in H D_{0}\left(\Omega_{0}\right)$ (see Maeda (1968), p. 18). If $G_{a}=\widetilde{G}_{a}-\widetilde{G}_{a^{*}}$ is the ordinary Green function for $\Omega_{0}$ with pole at $a$ then $U_{a}=2 \tilde{G}_{a^{*}}+2 \varphi_{n} W(a)\|W\|^{-2} W$ and $N_{a}=G_{a}+U_{a}$.

Corollary to THEOREM 2.1. If $\hat{a} \in \partial \Omega$ then the Kuramochi function on $\Omega_{0} \cup \partial \Omega \cup\{\infty\}$ corresponding to $\hat{a}$ is $N_{\hat{a}}=2 \widetilde{G}_{\hat{a}}+2 \varphi_{n} W(\hat{a})\|W\|^{-2} W$ and if $\hat{a}=\infty$ then $N_{\hat{a}}=2 \varphi_{n}\|W\|^{-2} W$.

Proof. A sequence $\left(a_{n}\right)$ in $\Omega$ which eventually leaves compact subsets of $\Omega$ is fundamental (or Cauchy) in the Kuramochi sense if and only if the sequence $\left(N_{a_{n}}\right.$ converges locally uniformly to a unique harmonic function on $\Omega_{0}$. According to our construction of $N_{a}$ for each $a \in \Omega_{0}$ it is clear that $\left(a_{n}\right)$ is (Kuramochi) fundamental if and only if $\left(a_{n}\right)$ converges to a point of $\partial \Omega \cup\{\infty\}$. Hence the Kuramochi boundary of $\Omega\left(\bmod K_{0}\right)$, denoted by $\Delta_{N}$, may be identified with $\partial \Omega \cup\{\infty\} . \quad / /$

REMARK 2.3. All Kuramochi boundary elements of $\Omega$ are extremal (or minimal) and any full-superharmonic function on $\Omega_{0}$ of potential type (see Maeda (1968), p. 17) can be uniquely represented as $N \mu(x)=\int N(x, y) d \mu(y)$ where $N$ is the Kuramochi kernel on $\left(\Omega_{0} \cup \Delta_{N}\right) \times\left(\Omega_{0} \cup \Delta_{N}\right)$ and $\mu$ is a Radon measure charging $\Omega_{0} \cup \Delta_{N}$.

LEMMA 2.2. If $\hat{a} \in \Delta_{N}$ (identified with $\partial \Omega \cup\{\infty\}$ ) and $E \subset \Omega_{0} \cup \Delta_{N}$ then $E \cup E^{*}$ is ordinary thin at $\hat{a}$ if $E$ is full-thin at $\hat{a}$ where $E^{*}$ is the reflection of $E$ about $\partial \Omega$.

Proof. If $E$ is full-thin at $\hat{a}$ and $\hat{a}$ is not an isolated point of $E \cup\{\hat{a}\}$ then there exists a Radon measure $\mu$ charging $\Omega_{0} \cup \Delta_{N}$ such that $N \mu \in \mathscr{P}$ and

$$
N \mu(\hat{a})<\liminf _{x \rightarrow a, x \in E} N \mu(x) \text {. }
$$

But $N \mu$ is the restriction to $\Omega_{0} \cup \Delta_{N}$ of a positive superharmonic function $S$ on $\Omega_{0} \cup\{\infty\}$ of the form

$$
S(x)=\tilde{G} v(x)+\lambda W(x)
$$


where $v=\left.\mu\right|_{\Omega_{0} \cup \partial \Omega}+\left(\left.\mu\right|_{\Omega_{0} \cup \Omega \Omega}\right)^{*}$ given that the star means a reflection about $\partial \Omega$ and $\lambda=\mu\{\infty\}+\varphi_{n}\|W\|^{-2}\left(\int_{\Omega_{0}} W(y) d v(y)\right)$. Hence $E$ as well as $E \cup E^{*}$ is ordinary thin at $\hat{a} \in \Delta_{N}$ because $S$ is symmetric about $\partial \Omega$ (see Brelot (1944), p. 313, when $\hat{a}=\infty$ ). The lemma follows. //

LEMmA 2.3. If $\hat{a} \in \Delta_{N}$ and $E \subset \Omega_{0} \cup \Delta_{N}$ such that $E$, and hence $E \cup E^{*}$, is ordinary thin at $\hat{a}$ then $E$ is full-thin at $\hat{a}$.

Proof. Case 1. $\hat{a} \in \partial \Omega$. There is a Green potential $\tilde{G} \mu$ of a mass distribution $\mu$ on $\Omega_{0} \cup \partial \Omega$ such that the total mass of $\mu$ is finite and

$$
\widetilde{G} \mu(a)<\liminf _{x \rightarrow a, x \in E} \tilde{G} \mu(x) .
$$

If $\mu^{*}$ is the reflection of $\mu$ about $\partial \Omega$ and $v=\mu+\mu *$ then

$$
\tilde{G} v(\hat{a})<\liminf _{x \rightarrow a, x \in E} \tilde{G} v(x)
$$

and since $\tilde{G} v$ is symmetric about $\partial \Omega$ it is clear that $E \cup E^{*}$ is ordinary thin at $\hat{a}$ if $E$ is. Now

$$
N \mu(x)=\tilde{G} v(x)+2 \varphi_{n}\|W\|^{-2}\left(\int_{\Omega_{0} \cup \partial \Omega} W(y) d \mu(y)\right) W(x) \quad\left(x \in \Omega_{0} \cup \Delta_{N}\right)
$$

which implies that $N \mu \in \mathscr{P}$ since $\mu\left(\Omega_{0} \cup \partial \Omega\right)<\infty$. Furthermore

$$
\liminf _{x \rightarrow a, x \in E} N \mu(x)-N \mu(\hat{a})=\liminf _{x \rightarrow a, x \in E} \tilde{G} v(x)-\tilde{G} v(\hat{a})>0
$$

which indicates that $E$ is full-thin at $\hat{a}$.

Proof. Case 2. $\hat{a}=\infty$. There exists a positive superharmonic function $S_{1}$ on $\widetilde{\Omega}_{0} \cup\{\infty\}$ of the form $S_{1}(x)=\tilde{G} \mu(x)+\lambda_{1} W(x)$ such that

$$
S_{1}(\hat{a})<\liminf _{x \rightarrow a, x \in E} S_{1}(x) .
$$

Without loss of generality we may assume that $\mu$ is supported by $\Omega_{0} \cup \Delta_{N}$. The reasoning in the proof of Lemma 2.1 indicates that $\widetilde{G}_{a}+\lambda_{a} W$ is superharmonic at $\infty$ if and only if $\lambda_{a} \geqslant \varphi_{n}\|W\|^{-2} W(a)$ and hence $S_{1}$ is superharmonic at $\infty$ if and only if

$$
\lambda_{1} \geqslant \varphi_{n}\|W\|^{-2}\left(\int_{\Omega_{0} \cup 2 \Omega} W(y) d \mu(y)\right) .
$$

Hence $N \mu \in \mathscr{P}$ and if $v=\mu+\mu *$ we again have

$$
\liminf _{x \rightarrow a, x \in E} N \mu(x)-N \mu(\hat{a})=\liminf _{x \rightarrow \hat{a}, x \in E} \tilde{G} v(x)-\tilde{G} v(\hat{a})>0
$$

which proves our lemma. // 
We can now combine Lemmas 2.2 and 2.3 to obtain

THEOREM 2.2. If $\hat{a} \in \Delta_{N}$ (identified with $\partial \Omega \cup\{\infty\}$ ) then $E \subset \Omega_{0} \cup \Delta_{N}$ is full-thin (or Kuramochi thin) at $\hat{a}$ if and only if $E \cup E^{*}$ is ordinary thin at $\hat{a}$ where $E$ is the reflection of $E$ about $\partial \Omega$.

REMARK 2.4. Since ordinary thin sets are not preserved by inversion maps about spheres the same is true for full-thin sets. This contrasts with the theory of minimally thin sets in the sense of Lelong-Ferrand and Naïm which are so preserved (see Brelot (1944), pp. 313-314, and Brelot (1971), chap. XVII). $E \subset \Omega_{0}$ is ordinary thin at $\infty$ if and only if $\hat{R}_{1}^{E}$ is a $\bar{G}$-potential of finite energy and hence $E$ has finite capacity in either the $\tilde{G}$ sense or the Newtonian sense.

\section{The case of the unit ball $\Omega \subset \mathbf{R}^{n}(n \geqslant 3)$}

If $x \in \mathbf{R}^{n}-\{0\}$ we let $x^{*}=\tau(x)=|x|^{-2} x$ denote its inverse with respect to the unit sphere $\partial \Omega$ where we also understand that $\tau$ interchanges 0 and the point at infinity. For convenience we choose $K_{0}=\left\{x \in \Omega:|x| \leqslant \frac{1}{2}\right\}, \Omega_{0}=\Omega-K_{0}, \Omega_{0}^{*}=\tau\left(\Omega_{0}\right)$ and $\Omega_{0}=\Omega_{0} \cup \partial \Omega \cup \Omega_{0}^{*}$ is the annulus $\left\{x \in \mathbf{R}^{n}: \frac{1}{2}<|x|<2\right\}$. If $a \in \Omega_{0}$ the Kuramochi function $N_{a}$ for $\Omega_{0}$ with pole at the point $a$ is a mixed Green function for $\Omega_{0}$ with pole at $a$ of order $|x-a|^{2-n}$ which vanishes continuously on $\partial K_{0}$ and whose (inner) normal derivative vanishes on $\partial \Omega$. We shall construct a positive superharmonic function $S_{a}$ on $\Omega_{0}$ whose restriction to $\Omega_{0} \cup \partial \Omega$ is $N_{a}$ even in the case where $a=\hat{a} \in \partial \Omega$. Such an extended function shall be obtained by applying to $N_{a}$ the Kelvin transformation (see Helms (1969), p. 36) about $\partial \Omega$. If $a \in \Omega_{0}$ the extended function $S_{a}$ is a positive superharmonic function on $\Omega_{0} \cup \Omega_{0}^{*}$ with poles at $a$ and $a^{*}$ and if $a=\hat{a} \in \partial \Omega$ then $S_{a}$ is harmonic on $\Omega_{0} \cup \Omega_{0}^{*}$. Furthermore, $S_{a}$ is continuous on $\Omega_{0}$ except at the poles (respectively pole) $a$ and $a^{*}$ (respectively $a=\hat{a}$ ) if $a \in \Omega_{0}$ (respectively $a=\hat{a} \in \partial \Omega$ ). More precisely we define the Kelvin transformation of $N_{a}$ to be

$$
V_{a}(x)=|x|^{2-n} N_{a}(\tau(x)) \quad \text { if } 1 \leqslant|x| \leqslant 2 .
$$

It is clear that $N_{a}$ and $V_{a}$ coincide on $\partial \Omega$ so that we may define $S_{a}$ on $\Omega_{0}$ such that $S_{a \mid \Omega_{0} \cup \Omega \Omega}=N_{a}$, and $S_{\Omega_{0} \cup \Omega \Omega}=V_{a}$. We know that $S_{a}$ is superharmonic on $\Omega_{0} \cup \Omega_{0}^{*}$ but it remains to demonstrate that $S_{a}$ is also superharmonic on $\partial \Omega$ as well. If $\hat{x} \in \partial \Omega, a \neq \hat{x}$, we shall denote the outer (respectively inner) normal derivative of $S_{a}$ at $\hat{x}$ by the symbol $\partial S_{a} / \partial v_{e}(\hat{x})\left(\right.$ respectively $\left.\partial S_{a} / \partial v_{i}(\hat{x})\right)$.

Lemma 3.1. If $\hat{x} \in \partial \Omega, a \neq \hat{x}$, then $\partial S_{a} / \partial v_{e}(\hat{x})=(2-n) N_{a}(\hat{x})$. 
ProOF. Let

$$
x \in \Omega_{0}, \quad r=|x|, \quad \hat{x}=\frac{x}{r}, \quad x^{*}=\frac{\hat{x}}{r}=\hat{x}+h \hat{x} \quad\left(h>0, \frac{1}{2} \leqslant r \leqslant 1\right) .
$$

Then $S_{a}(\hat{x}+h \hat{x})-S_{a}(\hat{x})=V_{a}\left(\mathrm{x}^{*}\right)-N_{a}(\hat{x})=|x|^{n-2} N_{a}(x)-N_{a}(\hat{x})$. Since $r=|x|$,

$$
h=\frac{1}{r}-1=\frac{1-r}{r}
$$

therefore

$$
\frac{S_{a}(\hat{x}+h \hat{x})-S_{a}(\hat{x})}{h}=\frac{r}{(1-r)}\left\{\left(r^{n-2}-1\right) N_{a}(r \hat{x})+\left(N_{a}(r \hat{x})-N_{a}(\hat{x})\right)\right\} .
$$

Now $h \rightarrow 0^{+}$if and only if $r \rightarrow 1^{-}$so that

$$
\frac{\partial S_{a}}{\partial v_{e}}(\hat{x})=(2-n) N_{a}(\hat{x})+\frac{\partial N_{a}}{\partial v_{i}}(\hat{x}) .
$$

Since

$$
\frac{\partial N_{a}}{\partial v_{i}}(\hat{x})=\frac{\partial S_{a}}{\partial v_{i}}(\hat{x})=0
$$

the lemma follows. //

REMARK 3.1. In the higher dimensional case (that is $n \geqslant 3$ ) we have

$$
\frac{\partial S_{a}}{\partial v_{i}}(\hat{x})+\frac{\partial S_{a}}{\partial v_{e}}(\hat{x})=(2-n) N_{a}(\hat{x})<0
$$

whenever $a \neq \hat{x}$. This introduces a complication that was not present in the twodimensional case where this quantity always vanished.

LEMMA 3.2. If $\hat{x}_{0} \in \partial \Omega, a \neq \hat{x}_{0}$ and $B_{\varepsilon}=\left\{x \in \mathbf{R}^{n}:\left|x-x_{0}\right|<\varepsilon\right\}$ then the outer flux of $S_{a}$ over $\partial B_{\varepsilon}$ is always negative if $\varepsilon>0$ is sufficiently small and therefore $S_{a}$ is superharmonic at $\hat{x}_{0}$ and hence on $\partial \Omega$.

ProOF. Since $S_{a}$ is harmonic on the region $\Omega_{0} \cap B_{\varepsilon}$ to which the divergence theorem is applicable we can employ Green's first identity to obtain

$$
\int_{\Omega_{0} \cap \hat{\partial} B_{e}}\left(\frac{\partial S_{a}}{\partial v_{e}}\right) d \sigma_{n-1}=\int_{B_{\varepsilon} \cap \partial \Omega}\left(\frac{\partial S_{a}}{\partial v_{i}}\right) d \sigma_{n-1} \text { (which vanishes in this case). }
$$

Similar reasoning can be applied to $S_{a \mid \Omega_{o}^{*} \cap B_{\varepsilon}}$ to obtain

$$
\int_{\Omega_{0}^{*} \cap \delta B_{t}}\left(\frac{\partial S_{a}}{\partial v_{e}}\right) d \sigma_{n-1}=\int_{B_{e} \cap \partial \Omega}\left(\frac{\partial S_{a}}{\partial v_{e}}\right) d \sigma_{n-1} .
$$

Since $\sigma_{n-1}\left(\partial B_{\varepsilon}\right)=\sigma_{n-1}\left(\Omega_{0} \cap \partial B_{\varepsilon}\right)+\sigma_{n-1}\left(\Omega_{0}^{*} \cap \partial B_{\varepsilon}\right)$ we can add (3.3) and (3.3)' to obtain 


$$
\int_{\hat{c} B_{e}}\left(\frac{\partial S_{a}}{\partial v_{e}}\right) d \sigma_{n-1}=\int_{B_{t} \cap \hat{\partial} \Omega}\left(\frac{\partial S_{a}}{\partial v_{i}}+\frac{\partial S_{a}}{\partial v_{e}}\right) d \sigma_{n-1}<0 .
$$

In order to see that $S_{a}$ is superharmonic at $\hat{x}_{0}$ we let $\mathscr{M}_{S_{a}}\left(\hat{x}_{0}\right)$ be the mean value of $S_{a}$ over the sphere $\partial B_{r}$ of centre $\hat{x}_{0}$ where $0 \leqslant r \leqslant \varepsilon$ with the understanding that $S_{a}\left(\hat{x}_{0}\right)=\mathscr{M}_{S_{a}}^{o}\left(\hat{x}_{0}\right)$.

Then

$$
\left.\sigma_{n-1}\left(\partial B_{1}\right) \mathscr{M}_{S_{a}}^{r}\left(\hat{x}_{0}\right)\right)=r^{1-n} \int_{\partial B_{r}} S_{a}\left(\hat{x}_{0}+r \hat{u}\right) d \sigma_{n-1}=\int_{\partial B_{1}} S_{a}\left(\hat{x}_{0}+r \hat{u}\right) d \sigma_{n-1}(\hat{u}),
$$

where $\hat{u}$ is designated as any point on $\partial B_{1}$. Furthermore, we can take the first derivative with respect to $r$ to obtain

$$
\begin{aligned}
\sigma_{n-1}\left(\partial B_{1}\right) \frac{d}{d r} \mathscr{M}_{S_{a}}\left(\hat{x}_{0}\right) & =\int_{\partial B_{1}} \frac{\partial}{\partial r} S_{a}\left(\hat{x}_{0}+r \hat{u}\right) d \sigma_{n-1}(\hat{u}) \\
& =r^{1-n} \int_{\partial B_{r}}\left(\frac{\partial S_{a}}{\partial v_{e}}\right) d \sigma_{n-1}<0 .
\end{aligned}
$$

It follows that $\mathscr{M}_{S_{a}}^{r}(\hat{x})$ is a decreasing function of $r$ on $[0, \varepsilon]$ so that $S_{a}\left(\hat{x}_{0}\right) \geqslant \mathscr{M}_{S_{a}}^{\varepsilon}\left(\hat{x}_{0}\right)$. Since $\hat{x}_{0} \in \partial \Omega$ is arbitrary it follows that $S_{a}$ is superharmonic on $\partial \Omega$ as well as on $\Omega_{0} \cup \Omega_{0}^{*}$. //

REMARK 3.2. If $a \in \partial \Omega$ we have only shown that $S_{a}$ is superharmonic on $\Omega_{0}-\{a\}$. Since $S_{a}$ has a pole at $a$, however, it is clear that $S_{a}$ is superharmonic at $a$ as well. It is also clear that $S_{a}$ is a Green potential on $\tilde{\Omega}_{0}$ of a mass distribution which charges only $\partial \Omega$ if $a \in \partial \Omega$ and charges $\partial \Omega \cup\{a\} \cup\left\{a^{*}\right\}$ if $a \in \Omega_{0}$. This results from the fact that $S_{a}$ is harmonic elsewhere on $\Omega_{0}$ and vanishes continuously on $\partial \widetilde{\Omega}_{0}$.

LeMma 3.3. If $a \in \Omega_{0} \cup \partial \Omega$ then

$$
S_{a}(x)=\tilde{G}_{a}(x)+|a|^{2-n} \tilde{G}_{a^{*}}(x)+K_{n} \int_{\partial \Omega} \tilde{G}(x, z) N_{a}(z) d \sigma_{n-1}(z),
$$

where $K_{n}$ is a nonnegative constant that depends only on the dimension $n$.

Proof. We first suppose that $a \in \Omega_{0}$. We assume that $N_{a}$ and $\tilde{G}_{a}$ both have poles at $a$ of the form $|x-a|^{2-n}$ so that the canonical measure for $S_{a}$ that is concentrated on $\{a\}$ is the unit measure. In order to find the canonical measure for $S_{a}$ that is concentrated on $\left\{a^{*}\right\}$ we find the Kelvin transformation of $G_{a}$ to be denoted by $\left(\tilde{G}_{a}\right)^{*}$. Now $\left(\tilde{G}_{a}\right)^{*}(x)=|x|^{2-n} \tilde{G}_{a}\left(x^{*}\right)$ is a harmonic function of $x$ on $\Omega_{0}-\left\{a^{*}\right\}$ and is therefore of the form $\gamma_{a} \widetilde{G}_{a^{*}}(x)$ where $\gamma_{a}$ is a constant to be determined. If we let $x=a$ and take advantage of the symmetry of the Green kernel $\tilde{G}$ we obtain $\gamma_{a}=|a|^{2-n}$ 
which is the total mass of the canonical measure for $S_{a}$ that is concentrated on $\left\{a^{*}\right\}$. By reasoning as in Kellogg(1929), p. 164, who treats the case $n=3$ we obtain the fact that the density function with respect to $\sigma_{n-1}$ of the canonical measure for $S_{a}$ that is concentrated on $\partial \Omega$ is a constant multiple of $\left(\partial S_{a} / \partial v_{e}+\partial S_{a} / \partial v_{i}\right)$ where the constant only depends on the dimension $n$. In light of Lemma 3.1 this density function can be written as $K_{n} N_{a}(z)$ for any $z \in \partial \Omega$ where $K_{n} \geqslant 0$. Therefore

$$
S_{a}(x)=\tilde{G}_{a}(x)+|a|^{2-n} \tilde{G}_{a^{*}}(x)+K_{n} \int_{\partial \Omega} \tilde{G}(x, z) N_{a}(z) d \sigma_{n-1}(z) \text { if } a \in \Omega_{0}
$$

and by a continuity argument we obtain

$$
S_{\hat{a}}(x)=2 \widetilde{G}_{\hat{a}}(x)+K_{n} \int_{\partial \Omega} \tilde{G}(x, z) N_{\hat{a}}(z) d \sigma_{n-1}(z) \quad \text { if } \hat{a} \in \hat{\partial} \Omega . \quad / /
$$

REMARK 3.3. By using Green's third identity one can compute the value of the constant $K_{n}$ to be $(n-2) / \varphi_{n}$ which vanishes when $n=2$ and equals $\left(\sigma_{n-1}\left(\partial B_{1}\right)\right)^{-1}$ when $n \geqslant 3$. The surface measure of the unit sphere, written as $\sigma_{n-1}\left(\partial B_{1}\right)$, is given in Landkof (1972), p. 18 , to be $2 \pi^{n / 2}(\Gamma(n / 2))^{-1}$. For example $K_{3}=(4 \pi)^{-1}$. We also remark that the ordinary Green function $G_{a}$ for $\Omega_{0}$ with pole at $a$ is $G_{a}=\tilde{G}_{a}-|a|^{2-n} \widetilde{G}_{a^{*}}$ restricted to $\Omega_{0}$. Again we can say that $N_{a}=G_{a}+U_{a}$ where $U_{a} \in H D_{0}\left(\Omega_{0}\right)$ such that the Dirichlet inner product $\left(U_{a}, u\right)=\varphi_{n} u(a)$ for every $u \in H D_{0}\left(\Omega_{0}\right)$. In this instance we can explicitly find

$$
U_{a}=2|a|^{2-n} \tilde{G}_{a^{*}}+K_{n} \int_{\partial \Omega} \tilde{G}(x, z) N_{a}(z) d \sigma_{n-1}(z)
$$

restricted to $\Omega_{0} \cup \partial \Omega$. We again identify the Kuramochi boundary $\Delta_{N}$ of $\Omega$ with $\partial \Omega$.

THEOREM 3.1. If $E \subset \Omega_{0} \cup \partial \Omega$ is full-thin at $\hat{x}_{0} \in \Delta_{N}$ then $E$ and hence $E \cup E^{*}$ is ordinary thin at $\hat{x}_{0}$.

PROOF. The Kuramochi kernel $N(x, y)$ is the restriction to $\left(\Omega_{0} \cup \partial \Omega\right) \times\left(\Omega_{0} \cup \partial \Omega\right)$ of the kernel $S(x, y)$ on $\Omega_{0} \times\left(\Omega_{0} \cup \partial \Omega\right)$ where

$$
S(x, y)=\widetilde{G}(x, y)+\widetilde{G}\left(x, y^{*}\right)|y|^{2-n}+K_{n} \int_{\partial \Omega} \tilde{G}(x, z) N(z, y) d \sigma_{n-1}(z) .
$$

If $\mu$ is a mass distribution on $\Omega_{0} \cup \partial \Omega$ so that $N \mu \neq \equiv+\infty$ then $N \mu$ is the restriction of the potential $S \mu$ to $\Omega_{0} \cup \partial \Omega$. Now

$$
S \mu(x)=\tilde{G} \mu(x)+(\tilde{G} \mu)^{*}(x)+K_{n} \int_{\partial \Omega} \tilde{G}(x, z) N \mu(z) d \sigma_{n-1}(z)
$$

which is the $\widetilde{G}$-potential of a mass distribution on $\Omega_{0}$. Furthermore, $S \mu$ is invariant under the Kelvin transformation so that if $E \subset \Omega_{0} \cup \partial \Omega$ then 


$$
\liminf _{x \rightarrow \hat{x}_{0}, x \in E} S \mu(x)=\liminf _{x \rightarrow \hat{x}_{0}, x \in E^{*}} S \mu(x),
$$

where $E^{*}=\tau(E)$. If $E \subset \Omega_{0} \cup \partial \Omega$ is full-thin at $\hat{x} \in \Delta_{N}$ then there exists a Kuramochi potential $N \mu$ of a mass distribution $\mu$ on $\Omega_{0} \cup \partial \Omega$ such that

$$
N \mu\left(\hat{x}_{0}\right)<\liminf _{x \rightarrow \hat{\Omega}_{0}, x \in E} N \mu(x) .
$$

Hence

$$
S \mu\left(\hat{x}_{0}\right)<\liminf _{x \rightarrow x_{0}, x \in E \cup E^{*}} S \mu(x)
$$

and since $S \mu$ is a $\bar{G}$-potential of a mass distribution on $\widetilde{\Omega}_{0}$ the theorem follows. $/ /$

REMARK 3.4. In order to prove the converse of Theorem 3.1 we shall require some estimates for the order of the pole at $\hat{a} \in \partial \Omega$ for $N_{\hat{a}}$. The next three lemmas will demonstrate that the order of the pole for $N_{\hat{a}}$ at $\hat{a}$ is a constant multiple of $|x-\hat{a}|^{2-n}$ and therefore coincides with the order of the pole for $\tilde{G}_{\hat{a}}$ at $\hat{a}$. If $f \geqslant 0$ and $g \geqslant 0$ on a set $X$ we shall say that $f \simeq g$ (on $X$ ) or $f$ is comparable to $g$ on $X$ provided that there exist constants $C_{1}>0, C_{2}>0$ (depending on $X$ in general) such that $C_{1} f \leqslant g \leqslant C_{2} f$ on $X$. We also let $f_{\alpha}(x)=|x|^{-\alpha}$ where $\alpha>0$ and notice that $f_{\alpha} \simeq k_{n-\alpha}$ where $k_{n-\alpha}$ is the M. Riesz kernel defined in $\mathbf{R}^{n}$ (see Landkof (1972), p. 43). We shall only include a proof for Lemma 3.4. Similar arguments can be used for the proofs of Lemmas 3.5 and 3.6 respectively.

LEMMA 3.4. If

$$
(x, y) \in \Omega_{0} \times \Omega_{0} \quad \text { and } f_{\alpha, \beta}(x, y)=\int_{\alpha \Omega} \frac{d \sigma_{n-1}(z)}{|x-z|^{\alpha}|z-y|^{\beta}},
$$

where $\alpha \geqslant 0, \beta>0$ and $\alpha+\beta+1<n$ then $f_{\alpha \beta}$ is bounded on $\widetilde{\Omega}_{0} \times \Omega_{0}$. The same result holds true if $|x-z|^{-\alpha}$ is replaced by $\max \{\log 1 /|x-z|, 0\}=\log ^{+} 1 /|x-z|$ provided that $0<\beta<n-1$.

ProOf. Let

$$
A_{1}=\{z \in \partial \Omega:|x-z| \leqslant|z-y|\}, \quad A_{2}=\{z \in \partial \Omega:|x-z|>|z-y|\} .
$$

Then

$$
f_{\alpha, \beta}(x, y) \leqslant \int_{A_{1}} \frac{d \sigma_{n-1}(z)}{|x-z|^{\alpha+\beta}}+\int_{A_{2}} \frac{d \sigma_{n-1}(z)}{|y-z|^{\alpha+\beta}} .
$$

In relation to $\mathbf{R}^{n}, f_{\alpha}$ is comparable to the M. Riesz kernel $k_{n-\alpha}$ (see Landkof (1972), p. 43) and therefore

$$
f_{\alpha, \beta}(x, y) \leqslant\left(k_{n-(\alpha+\beta)}^{*} \sigma_{n-1}\right)(x)+\left(k_{n-(\alpha+\beta)}^{*} \sigma_{n-1}\right)(y) .
$$


Since the surface measure $\sigma_{n-1}$ is locally absolutely continuous with respect to $(n-1)$-dimensional Lebesgue measure on the tangent space at any point $\hat{x}_{0} \in \partial \Omega$ such that the density function is bounded, therefore

$$
\left(k_{n-(\alpha+\beta)}^{*} \sigma_{n-1}\right)\left(x_{0}\right)=\int_{\partial B} \frac{d \sigma_{n-1}(z)}{\left|\hat{x}_{0}-z\right|^{\alpha+\beta}} \simeq \int_{0}^{\frac{1}{2}} \frac{r^{n-2} d r}{r^{\alpha+\beta}}
$$

which converges because $\alpha+\beta+2-n<1$. Hence the potential $k_{n-(\alpha+\beta)}^{*} \sigma_{n-1}$ is bounded on $\partial \Omega$ and by the weak maximum principle (see Landkof (1972), p. 66, Theorem 1.5) it is bounded on all of $\mathbf{R}^{n}$ and in particular on $\widetilde{\Omega}_{0}$. This implies that $f_{\alpha, \beta}$ is bounded on $\widetilde{\Omega}_{0} \times \widetilde{\Omega}_{0}$. For the second part we notice that for any $\alpha>0$ there exists $\delta$ (depending on $\alpha$ ) such that $\log ^{+}(1 / r) \leqslant r^{-\alpha}$ on $(0, \delta]$. Since $\beta+1<n$ we can define $2 \alpha>0$ such that $2 \alpha+\beta+1=n$ and therefore $\alpha+\beta+1<n$. If $x \in \widetilde{\Omega}_{0}$ is given and $B_{\delta}$ is the ball of centre $x$ and radius $\delta$ then

$$
\begin{aligned}
& \int_{\partial \Omega}\left(\log ^{+} \frac{1}{|x-z|}\right)|z-y|^{-\beta} d \sigma_{n-1}(z) \\
& \leqslant \int_{\partial \Omega \cap B_{\delta}}|x-z|^{-\alpha}|z-y|^{-\beta} d \sigma_{n-1}(z)+\delta^{-\alpha} \int_{\partial \Omega-B_{d}}|z-y|^{-\beta} d \sigma_{n-1}(z) .
\end{aligned}
$$

The lemma follows because both terms on the right of the inequality are bounded.

LeMMA 3.5. If $\alpha>0, \beta>0, \alpha+\beta+1=n$ and $y \in \partial \Omega$ then

$$
f_{\alpha, \beta}(x, y)=\int_{\partial \Omega}|x-z|^{-\alpha}|y-z|^{-\beta} d \sigma_{n-1}(z) \simeq \log \frac{1}{|x-y|}
$$

provided that $x \in \widetilde{\Omega}_{0}$ such that $|x-y| \leqslant \frac{1}{2}$.

Lemma 3.6. If $\alpha>0, \beta>0, \max \{\alpha, \beta\}<n-1$ and $\alpha+\beta+1>n$ then

$$
f_{\alpha, \beta}(x, y) \simeq \frac{1}{|x-y|^{\alpha+\beta+1-n}}
$$

if $y \in \partial \Omega$ and $x \in \widetilde{\Omega}_{0}$.

REMARK 3.5. In the last three lemmas we have demonstrated that, in general, the growth order of $f_{\alpha, \beta}$ is strictly less than that of $f_{\alpha}$ or $f_{\beta}$. In order to find the growth order of $N_{\hat{a}}$ at its pole $\hat{a} \in \partial \Omega$ we initially let $\alpha=\beta=n-2$ and then apply Lemma 3.5 if $n=3$, because $\alpha+\beta+1=n$, or Lemma 3.6 if $n \geqslant 4$, because $\alpha+\beta+1-n=n-3>0$.

Lemma 3.7. If $\hat{a} \in \partial \Omega$ then $N(x, \hat{a}) \simeq|x-\hat{a}|^{2-n}$ in a suitable neighbourhood of $\hat{a}$ in $\Omega_{0} \cup \partial \Omega$. 
ProOF. Since $\tilde{G}(x, \hat{a}) \simeq|x-\hat{a}|^{2-n}$ in a neighbourhood of $\hat{a}$ and since $N(x, \hat{a}) \geqslant 2 \widetilde{G}(x, \hat{a})$ (see Lemma 3.3) we have the inequality going one way. In order to obtain the inequality in the other direction we employ an iterated kernel theory (see Kellogg (1929), p. 288). Let $\widetilde{G}=\widetilde{G}_{0}$ on $\widetilde{\Omega}_{0} \times \widetilde{\Omega}_{0}$ and then define

$$
\tilde{G}_{j+1}(x, y)=\int_{\partial \Omega} \tilde{G}(x, z) \tilde{G}_{j}(z, y) d \sigma_{n-1}(z) \text { for all } j \geqslant 0 .
$$

From Lemma 3.3 we have

$$
\begin{aligned}
N(x, \hat{a})= & 2 \tilde{G}(x, \hat{a})+K_{n} \int_{\partial \Omega} \tilde{G}(x, z) N(z, \hat{a}) d \sigma_{n-1}(z) \\
=2 \tilde{G}(x, a) & +K_{n} \int_{\partial \Omega} \tilde{G}(x, z) \\
& \times\left\{2 \tilde{G}(z, \hat{a})+K_{n} \int_{\partial \Omega} \tilde{G}\left(x, z^{\prime}\right) N\left(z^{\prime}, \hat{a}\right) d \sigma_{n-1}\left(z^{\prime}\right)\right\} d \sigma_{n-1}(z) \\
\simeq & \tilde{G}_{0}(x, \hat{a})+\tilde{G}_{1}(x, \hat{a})+\int_{\partial \Omega} \tilde{G}_{1}\left(x, z^{\prime}\right) N\left(z^{\prime}, \hat{a}\right) d \sigma_{n-1}\left(z^{\prime}\right) .
\end{aligned}
$$

By an inductive argument we obtain

$$
N(x, \hat{a}) \simeq \sum_{j=0}^{n-1} \tilde{G}_{j}(x, \hat{a})+\int_{\partial \Omega} \tilde{G}_{n-1}(x, z) N(z, \hat{a}) d \sigma_{n-1}(z) .
$$

If $0 \leqslant j \leqslant n-3$ then $\tilde{G}_{j}(x, \hat{a}) \simeq|x-a|^{j+2-n}$ by an inductive application of Lemma 3.6. If $j=n-2$ so that $\alpha=1, \beta=n-2$ then $\alpha+\beta+1=n$ so that we can apply Lemma 3.5 in order to obtain $\widetilde{G}_{n-2}(x, \hat{a}) \simeq \log (1 /|x-\hat{a}|)$. If $j=n-1$ then we apply the second part of Lemma 3.4 to obtain the fact that $\tilde{G}_{n-1}$ is bounded on $\Omega_{0} \cup \partial \Omega$ by a constant $M>0$. Therefore

$$
N(x, \hat{a}) \leqslant \text { const. }|x-\hat{a}|^{2-n}+M \int_{\partial \Omega} N(z, \hat{a}) d \sigma_{n-1}(z) .
$$

Since the mass distribution on $\partial \Omega$ whose density function is $N(z, \hat{a})$ with respect to $\sigma_{n-1}$ has compact support therefore its total mass must be finite and therefore $N(x, \hat{a}) \leqslant$ const. $|x-\hat{a}|^{2-n}$ in some neighbourhood of $\hat{a}$ in $\Omega_{0} \cup \partial \Omega$. The lemma follows. //

THEOREM 3.2. If $E \subset \Omega_{0} \cup \partial \Omega$ is ordinary thin at $\hat{x}_{0} \in \partial \Omega$ then $E$ is full-thin at $\hat{x}_{0} \in \Delta_{N}$.

Proof. If $E$ is ordinary thin at $\hat{x}_{0}$ then there exists a $\tilde{G}$-potential of a mass distribution $\mu$ whose compact support is contained in $\Omega_{0} \cup \partial \Omega$ such that 


$$
\tilde{G} \mu\left(\hat{x}_{0}\right)<+\infty \text { and } \lim _{x \rightarrow x_{0}, x \in E} \tilde{G} \mu(x)=+\infty .
$$

Since $\tilde{G} \leqslant N$ on $\left(\Omega_{0} \cup \partial \Omega\right) \times\left(\Omega_{0} \cup \partial \Omega\right)$ therefore $\tilde{G} \mu(x) \leqslant N \mu(x)$ everywhere on $\Omega_{0} \cup \partial \Omega$. Therefore

$$
\lim _{x \rightarrow x_{0}, x \in E} N \mu(x)=+\infty
$$

and it remains to show that $N \mu\left(\hat{x}_{0}\right)<+\infty$. If $B_{\delta}$ is a suitable $\delta$-neighbourhood of $\hat{x}_{0}$ in $\Omega_{0} \cup \partial \Omega$ then

$$
N \mu\left(\hat{x}_{0}\right)=\int_{B_{\delta}} N\left(\hat{x}_{0}, y\right) d \mu(y)+\int_{\Omega-B_{d}} N\left(\hat{x}_{0}, y\right) d \mu(y) .
$$

In $B_{\delta}$ we have $N\left(\hat{x}_{0}, y\right) \simeq\left|\hat{x}_{0}-y\right|^{2-n} \simeq \tilde{G}\left(\hat{x}_{0}, y\right)$ by Lemma 3.7 and in $\Omega-B_{\delta}, N\left(\hat{x}_{0}, y\right)$ is bounded by a constant $M_{\delta}>0$. Therefore

$$
N \mu\left(\hat{x}_{0}\right) \leqslant \tilde{G} \mu\left(\hat{x}_{0}\right)+M_{\delta} \mu\left(\Omega_{0} \cup \partial \Omega\right)<+\infty
$$

which proves our theorem.

We now combine Theorems 3.1 and 3.2 to obtain our main result for the unit ball.

THEOREM 3.3. If $E \subset \Omega_{0} \cup \partial \Omega$ and $\hat{x}_{0} \in \partial \Omega$ then $E \cup E^{*}$ is ordinary thin at $\hat{x}_{0}$ if and only if $E$ is full-thin at $\hat{x}_{0} \in \Delta_{N}$.

\section{References}

M. Brelot (1944), 'Sur le role du point à l'infini dans la théorie des fonctions harmoniques', Ann. Sci. École Norm. Sup. 61, 301-332.

M. Brelot (1953), 'Etude et extensions du principe de Dirichlet', Ann. Inst. Fourier (Grenoble) 5, 371-419.

M. Brelot (1971), On topologies and boundaries in potential theory (Lecture Notes in Mathematics 175, Springer-Verlag, Berlin and New York).

C. Constantinescu and A. Cornea (1963), Ideale Ränder Riemannscher Flächen (Springer-Verlag (Ergeb. Math. Bd. 32), Berlin).

L. L. Helms (1969), Introduction to potential theory (Wiley-Interscience, New York).

J. S. Hwang and H. L. Jackson (1978), 'Some results on Kuramochi thin sets', An. Acad. Brasil. Ci. 50 (4), 441-445.

O. D. Kellogg (1929), Foundations of modern potential theory (Springer, Berlin).

N. S. Landkof (1972), Foundations of modern potential theory (Springer-Verlag (Grund. Math. Bd. 180), Berlin, New York).

F. Y. Maeda (1964), 'Notes on Green lines and Kuramochi boundary of a Green space', J. Sci. Hiroshima Univ. Ser. A-1 28, 59-66.

F. Y. Maeda (1966), 'Axiomatic treatment of full-superharmonic functions', J. Sci. Hiroshima Univ. Ser. A-1 30, 197-215.

F. Y. Maeda, M. Ohtsuka et al. (1968), Kuramochi boundaries of Riemann surfaces (Lecture Notes in Mathematics 58, Springer-Verlag, Berlin and New York).

M. Ohtsuka (1964), 'An elementary introduction of Kuramochi boundary', J. Sci. Hiroshima Univ. Ser. A-1 28, 271-299. 
Department of Mathematics McMaster University

Hamilton, Ontario

Canada
Centre de Recherches Mathématiques

and Université de Montréal

Montréal, Québec

Canada

also

Department of Pure Mathematics

Australian National University

Canberra, A.C.T. 2600

Australia 